\title{
Electronic Structure And Dynamics Of Higher-Lying Excited States In Light Harvesting Complex 1 From Rhodobacter sphaeroides
}

\author{
Peter D. Dahlberg ${ }^{1, \ddagger}$, Po-Chieh Ting ${ }^{2, \ddagger}$, Sara C. Massey ${ }^{2}$, Elizabeth C. Martin ${ }^{3}$, C. Neil \\ Hunter $^{3}$, and Gregory S. Engel ${ }^{2,}$ \\ ${ }^{1}$ Graduate Program in the Biophysical Sciences, Institute for Biophysical Dynamics, and the \\ James Franck Institute, The University of Chicago, Chicago, IL 60637 \\ 2Department of Chemistry, Institute for Biophysical Dynamics, and the James Franck Institute, \\ The University of Chicago, Chicago, IL 60637 \\ ${ }^{3}$ Department of Molecular Biology and Biotechnology, University of Sheffield, Firth Court, Western \\ Bank, Sheffield S10 2TN, UK
}

\begin{abstract}
Light harvesting in photosynthetic organisms involves efficient transfer of energy from peripheral antenna complexes to core antenna complexes, and ultimately to the reaction center where charge separation drives downstream photosynthetic processes. Antenna complexes contain many strongly coupled chromophores, which complicates analysis of their electronic structure. Twodimensional electronic spectroscopy (2DES) provides information on energetic coupling and ultrafast energy transfer dynamics, making the technique well suited for the study of photosynthetic antennae. Here, we present 2DES results on excited state properties and dynamics of a core antenna complex, light harvesting complex 1 (LH1), embedded in the photosynthetic membrane of Rhodobacter sphaeroides. The experiment reveals weakly allowed higher-lying excited states in LH1 at $770 \mathrm{~nm}$, which transfer energy to the strongly allowed states at $875 \mathrm{~nm}$ with a lifetime of $40 \mathrm{fs}$. The presence of higher-lying excited states is in agreement with effective Hamiltonians constructed using parameters from crystal structures and atomic force microscopy (AFM) studies. The energy transfer dynamics between the higher- and lower-lying excited states agree with Redfield theory calculations.
\end{abstract}

\section{Graphical abstract}

\footnotetext{
"Corresponding Author E-mail and phone: gsengel@uchicago.edu (773-704-0818).

¥Authors acknowledge equal contributions to the work

Supporting Information: Supplementary tables, figures, and a discussion of the fitting procedure and LH2 are presented in the supporting information. This material is available free of charge via the internet at http://pubs.acs.org.
} 


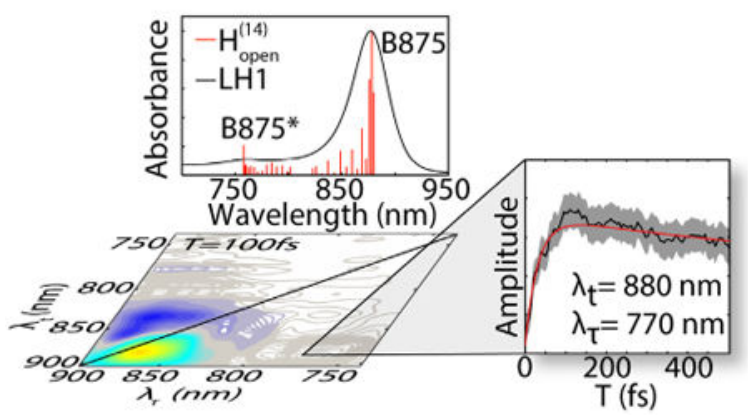

\section{Introduction}

Photosynthetic organisms have developed a nearly universal strategy in light harvesting, by which they spatially separate the absorption of solar energy and the use of that energy to generate separated charges. ${ }^{1}$ Rapid energy transfer from the site of absorption to the site of charge separation is crucial for efficient solar harvesting. In photosynthetic organisms, the absorption and charge separation sites can be separated by nearly one hundred nanometers, yet transfer occurs on the order of tens of picoseconds and with near unity quantum efficiency in low light. ${ }^{2-3}$ To accomplish this feat, they employ specialized pigment-proteins known as antenna complexes to absorb solar energy. Antenna complexes are packed with strongly coupled chromophores. This strong coupling leads to delocalized states with strong transition dipoles and a complex electronic structure that is essential for energy transfer. In this manuscript, we employ two-dimensional electronic spectroscopy (2DES) to observe the electronic structure and dynamics of light harvesting complex 1 (LH1) from Rhodobacter sphaeroides ( $R$. sphaeroides) embedded in its native photosynthetic membrane.

R. sphaeroides is a purple bacterium known for its near unity quantum efficiency ${ }^{3}$ and highly symmetric antenna complexes. $R$. sphaeroides has two types of antenna complexes: a peripheral complex, light harvesting complex 2 (LH2) and a core complex, light harvesting complex 1, that surrounds the reaction center (RC). An 8- $\AA$ resolution structure of an RCLH1-PufX dimer was obtained from a combination of X-ray crystallography and cryoelectron microscopy. ${ }^{4-5}$ This structure reveals an S-shaped array of chromophores that intertwines around two RCs. The bacteriochlorophyll (BChl) within the LH1 complex absorb maximally at $875 \mathrm{~nm}$, and are often designated as B875; they share many characteristics with the $\mathrm{B} 850$ ring of $\mathrm{BChl}$ in LH2. The RC-LH1-PufX complex exists in $R$. sphaeroides as both a monomer and a dimer, but the dimer is the dominant structure in vivo. Each monomer in the dimer contains $28 \mathrm{BChl}$ a molecules bound in alternating $\mathrm{a}$ - and $\beta$ polypeptides (Figure 1). ${ }^{4}$ The electronic coupling between BChl $a$ in LH1 is well approximated by dipolar coupling except in the case of nearest neighbors where the $\mathrm{Mg}-\mathrm{Mg}$ distance is on the order of $10 \AA .{ }^{4}$ The coupling strength of nearest neighbors is estimated to be a few hundred wavenumbers. ${ }^{6-7}$ This strong coupling leads to the delocalization of excitations across BChl $a$ and the existence of higher-lying excited states. A special pair of BChl $a$ embedded in each RC absorbs at $870 \mathrm{~nm}$ and accepts excitations from LH1 ${ }^{8-9}$ The LH1 complex used in this manuscript was obtained from a mutant of $R$. sphaeroides that lacked LH2, PufX, and the RC. In the absence of PufX and the RC, the predominant 
structure of the membrane-embedded LH1 is likely a closed, but empty ring. ${ }^{10-11}$ Highresolution AFM topographs of membrane-embedded LH1-only complexes showed that they are $9.9 \pm 0.7 \mathrm{~nm}$ in diameter, smaller than the $11.2 \pm 0.6 \mathrm{~nm}$ measured for monomeric RCLH1 core complexes with $16 \mathrm{a}_{1} \beta_{1} \mathrm{BChl}_{2}$ LH1 subunits. ${ }^{12}$ Accordingly, the LH1-only complex was assigned to be a ring of $15 \mathrm{a}_{1} \beta_{1} \mathrm{BChl}_{2}$ subunits. ${ }^{11}$

A closed ring, unlike an open ring, has an additional circular symmetry that leads to a series of pairwise degenerate electronic states. The manifold of states in a closed ring of LH1 adopts a labeling scheme to reflect these degeneracies. The lowest and highest energy states are the only non-degenerate states and, in the case of an LH1, are composed of 15 $a_{1} \beta_{1} B C h l_{2}$ subunits; these states are labeled $k=0$ and $k=15$. All other states are pairwise degenerate and are labeled $k= \pm 1 \ldots \pm 14$. In a perfectly symmetric monomeric ring, the transition dipole for most excited states becomes vanishingly small, so the only states with finite transition dipole strength are $\mathrm{k}= \pm 1$ and $\mathrm{k}= \pm 14$, with $\mathrm{k}= \pm 1$ being the dominant transition dipole. However, in nature, this perfect circular symmetry is never realized due to disorder. In the dimeric RC-LH1-PufX complex, the "S" shape breaks the circular symmetry and therefore the degeneracies. Observation of the higher-lying excited states and their lifetime in the photosynthetic membrane can inform on the nearest-neighbor coupling strengths, site energies and coupling to the bath, thus providing great insight into the electronic structure of LH1 in vivo.

Previous ultrafast spectroscopy studies have been performed on detergent isolated preparations of LH1. Fluorescence anisotropy data suggest the energy transfer between BChl a molecules occurs on $110 \mathrm{fs}$ and $400 \mathrm{fs}$ timescales. ${ }^{13}$ Three-photon echo peak shift, pumpprobe, and transient grating experiments resolved four dominant vibrational modes and two decay lifetimes. ${ }^{14-15}$ Pump-dump-probe and pump-probe experiments have characterized the exciton equilibration between the low-energy states as taking place on a $100 \mathrm{fs}$ timescale. ${ }^{16-17}$ To date, none of these ultrafast studies have analyzed simultaneously the higher-lying excited states and their coupling to the strongly allowed $k= \pm 1$ states. In this paper, we present a 2DES waiting time series of monomeric LH1-only complexes embedded in the photosynthetic membrane from $R$. sphaeroides. We find evidence of higher-lying excited states and resolve waiting time dynamics. We use this information to refine the site energies and nearest-neighbor couplings within the B875 band of chromophores and find good agreement between calculated and experimentally observed energy transfer rates.

\section{Experimental Methods}

\section{Preparation of LH1-Only Membranes}

A mutant containing only LH1 complexes, designated L3 ( $\triangle$ puc1BA $\triangle p u f L M X)$, was created by genomic deletion of puc1BA and pufLMX genes using the methodology described in Mothersole et al. ${ }^{18}$ The cells were cultured semi-aerobically in the dark at $30{ }^{\circ} \mathrm{C}$. LH1-only membranes were obtained by disrupting the cells using a French press at 14,000 PSI. A slow spin was performed (12,000 RPM JA 30.STI for 20 minutes) to remove large cellular debris. The supernatant was diluted to an optical density of 0.3 at $875 \mathrm{~nm}$ in a 200- $\mu$ m path length quartz cell (Starna Cells Inc.), which was used for analysis. 


\section{Two-Dimensional Electronic Spectroscopy}

Two-dimensional electronic spectroscopy correlates excitation energies $\left(\lambda_{\tau}\right)$ and stimulated emission/ground state bleach/excited state absorption energies $\left(\lambda_{t}\right)$ as a function of an ultrafast time delay. Spectrally and temporally resolved signals acquired from this spectroscopic technique are beneficial for determining the electronic structure of systems with dense absorption spectra, such as those of antenna complexes. ${ }^{19}$ The theory and experimental design are described elsewhere, ${ }^{20-24}$ here, the working principle and information content of a 2DES spectrum is summarized. 2DES is a third-order optical technique involving three ultrafast laser pulses interacting with a sample. The third-order polarization, created by the three light-matter interactions, generates a photon echo signal in a phase-matched direction. The signal is heterodyned with a fourth pulse known as the local oscillator and is spectrally resolved in a spectrometer, and the heterodyned signal is collected on a camera. A 2DES spectrum is a frequency-frequency correlation map, acquiring extra spectral resolution by Fourier transforming across the coherence time ( $\tau$, time delay between pulses 1 and 2) axis. 2DES spectra are collected as a function of the waiting time delay (T, time delay between pulses 2 and 3). 2DES has advantages of both spectral and temporal resolution, correlating energy transfer between excited states with different energies while obtaining lifetimes of excited states.

2DES spectra in this study were acquired using the GRadient-Assisted Photon Echo Spectroscopy (GRAPES) technique. ${ }^{25-28}$ A Ti:Sapphire regenerative amplifier (Coherent Inc.) was used to generate a $30 \mathrm{fs}$ FWHM pulse centered at $800 \mathrm{~nm}$ with a $5 \mathrm{kHz}$ repetition rate and a power of $2 \mathrm{~W}$. The pulse was focused through argon gas (4 psi) and then again through air to obtain a $\sim 140 \mathrm{~nm}$ broadband pulse. An SLM-based pulse shaper (Biophotonics Solutions) compressed the pulse to $15 \mathrm{fs}$ and shaped the spectrum producing the excitation spectrum shown in Figure 1, which was sufficiently broad to excite both the low energy and high energy states simultaneously. The power for pulses 1-3 was attenuated to $33 \mu \mathrm{J} / \mathrm{cm}^{2}\left(1.37 \times 10^{14}\right.$ photons $\left./ \mathrm{cm}^{2}\right)$. Pulse 4 was attenuated an additional 2 orders of magnitude. The coherence times were sampled from 0 to $200 \mathrm{fs}$ in $0.9 \mathrm{fs}$ steps using the gradient-assisted method. The echo signal was spectrally resolved and heterodyne detected on a high-speed CMOS camera (Phantom Miro M310) triggered at $1 / 100^{\text {th }}$ the repetition rate of the regenerative amplifier. The spectral phase was determined using separately acquired pump-probe spectra, see Figure S1. ${ }^{29}$ To extract timescales of waiting time dynamics, each waiting time trace in the phased data was fit to a model function with a biexponential decay plus an offset to recover both early-time dynamics and vibrational relaxations. The residuals from curve fitting at each point in the 2DES map were Fourier transformed to obtain information on vibrational frequencies associated with waiting time dynamics.

\section{Effective Hamiltonian of LH1}

Model Hamiltonians were constructed to reproduce the experimental results and revise physical parameters such as nearest neighbor coupling, site energies, and spectral density. Our sample contains a mixture of structures containing 14,15 , and $16 \alpha_{1} \beta_{1} B C h l_{2}$ subunits in both open and closed ring configurations. However, prior AFM studies indicate that the most prevalent structure of LH1 in the absence of PufX and the RC is a closed ring of 15 
$a_{1} \beta_{1} B_{C h l}$ subunits. ${ }^{10-11}$ Therefore, we constructed three separate effective Hamiltonians to account for the ill-defined structure of monomeric LH1 in the absence of PufX and the RC.

The first Hamiltonian, $\mathrm{H}_{\text {closed }}^{(15)}$, describes a closed ring of 15 subunits. Because no crystal structures exist with this geometry, a model was constructed with 30 equally spaced BChl a in the $\mathrm{XY}$ plane arranged in a ring with a radius of $5 \mathrm{~nm}$. The $\mathrm{Q}_{\mathrm{y}}$ dipole orientations were alternated between pointing at $203^{\circ}$ and $21^{\circ}$ in the XY plane. The orientation of the dipoles was taken from the average over the first 20 chromophores in the dimeric crystal structure starting from one end of the $\mathrm{S}$-shaped band. ${ }^{4}$ The site energies were made to alternate between $12150 \mathrm{~cm}^{-1}(823 \mathrm{~nm})$ and $12450 \mathrm{~cm}^{-1}(803 \mathrm{~nm})$ and the nearest neighbor coupling was approximated as $435 \mathrm{~cm}^{-1}$. These values were comparable to previous studies and reproduced features in the absorption spectra. ${ }^{6}$ The coupling for all other chromophores was calculated assuming purely dipolar interaction. A dipole strength of 8.3 Debye was adopted for all BChl a following the calculations in §ener et al. ${ }^{6}$ The second Hamiltonian, $\mathrm{H}_{\mathrm{open}}^{(14)}$, was calculated in the same manner except the distances and orientations of chromophores were taken directly from one half of the LH1 in the dimeric RC-LH1-PufX crystal structure shown in Figure 1 which consists of a nearly-closed ring of $14 a_{1} \beta_{1}$ BChl $_{2}$ LH1 subunits. ${ }^{9}$ The third Hamiltonian, $\mathrm{H}_{\text {closed }}^{(16)}$, was constructed in the same manner using the monomeric RC-LH1 structure from a similar organism, Thermochromatium tepidum. ${ }^{30}$ This $3.0 \AA$ resolution structure has a closed ring of $16 a_{1} \beta_{1} \mathrm{BChl}_{2}$ subunits that is slightly elliptical. The ellipticity breaks some of the degeneracies that arise from a closed ring.

The eigenstate energies and dipole strengths for $\mathrm{H}_{\text {open }}^{(14)}, \mathrm{H}_{\text {closed }}^{(15)}$, and $\mathrm{H}_{\text {closed }}^{(16)}$ are listed in Table S1, and stick spectra for all the Hamiltonians can be seen in Figure 2 along with the linear absorption spectrum of LH1-only membranes after the removal of Mie scatter. A comparison of the stick spectra to a 77 Kelvin absorption spectrum is made in SI Figures S2 and S3. All three Hamiltonians give rise to a strongly allowed band of excitonic states at low energy around $875 \mathrm{~nm}$. This feature is the well-known B875 absorption band of LH1. In the cases of $\mathrm{H}_{\text {closed }}^{(15)}$, and $\mathrm{H}_{\text {closed }}^{(16)}$, the transition dipole strength is carried almost exclusively by the $\mathrm{k} \pm 1$ states that have a transition energy of $11443 / 11401 \mathrm{~cm}^{-1}(874 / 877 \mathrm{~nm}) . \mathrm{H}_{\text {open }}^{(14)}$ provides a similar electronic structure, with the majority of the oscillator strength being carried by the three lowest energy states, E1-3, with transition energies of 11396-11445 $\mathrm{cm}^{-1}$ (877-874 nm). All three Hamiltonians also produce weak higher-lying excited states with transition energies at approximately $13195 \mathrm{~cm}^{-1}(760 \mathrm{~nm})$. These higher-lying excited states, in analogy to the higher-lying B850* states in $\mathrm{LH} 2$, will be referred to as B875*. The absorption spectrum is mirrored in the excitation fluorescence spectrum (Figure S4), indicating electronic coupling between the B875* states and the B875 states. Without further analysis, it would be difficult to separate this absorption feature from free BChl $a$ in the sample, which absorbs at $770 \mathrm{~nm}$ and overlaps B875*, or from vibrationally excited B875 states. Both free BChl $a$ as well as vibrationally excited states would likely relax to the B875 states before fluorescing. Therefore, the assignment of this feature is not conclusive based on linear absorption or fluorescence spectra alone and we therefore employ to 2DES and Redfield theory for an unambiguous assignment. 


\section{Results and Discussion}

The real portion of the phased rephasing 2DES spectra of LH1-only membranes at early waiting times, shown in Figure 3, reveal more features than the linear absorption spectrum. The strongest feature in the phased 2DES spectra appears as the near-diagonal peak at $\lambda_{\tau}=$ $870 \mathrm{~nm}, \lambda_{\mathrm{t}}=880 \mathrm{~nm}$. The offset from the diagonal is likely due to overlapping excited state absorption and stimulated emission. The absolute-valued spectra for the same waiting times are shown in Figure S5 and show a peak centered at $\lambda_{\tau}=875 \mathrm{~nm}$ for more details on the fitting procedure, $\lambda_{\mathrm{t}}=875 \mathrm{~nm}$. A regression of the phased data to a bi-exponential function yields two time constants of $\tau_{1}=55 \pm 2$ fs and $\tau_{2}=1130 \pm 26$ fs (Figure S6). Similar dynamics have been reported previously in LH1 and in the B850 ring from $\mathrm{LH} 2$ and have been attributed to vibrational relaxation, protein reorganization and spectral diffusion. ${ }^{14}$

A diagonal peak near $\lambda_{\tau}=\lambda_{t}=770 \mathrm{~nm}$ corresponding to $\mathrm{B} 875^{*}$ is not visible due to its low intensity relative to the B875 diagonal peak. In third-order experiments, the signal intensity scales with the fourth power of transition dipoles, whereas a signal in first-order experiments, such as that in a linear absorption spectrum, scales with the square of transition dipoles. Based on the linear absorption spectrum, we expect the relative strength of the B875* diagonal peak to be $\sim(0.05)^{2}$, or $0.25 \%$, of the intensity at the B875 diagonal peak, which is too weak to be observed. The signal intensity of a cross peak in a 2DES spectrum is the result of two interactions with each of the corresponding diagonal features. Based on the linear absorption spectrum, the intensity at $\lambda_{\tau}=770 \mathrm{~nm}$ and $\lambda_{t}=880 \mathrm{~nm}$ would be $5 \%$ of the B875 diagonal feature if energy transferred from B875* to B875. The cross peak observed between $\lambda_{\tau}=770 \mathrm{~nm}$ and $\lambda_{\mathrm{t}}=880 \mathrm{~nm}$ has a maximum of 0.023 in the normalized spectra (Figure 4 and Figure S7).

The position and amplitude of the cross peak indicate that the states observed in the linear absorption spectrum around $770 \mathrm{~nm}$ are transferring excitations to B875. Nevertheless, the existence of coupling and transfer could be due to vibrationally excited B875 states or free $\mathrm{BChl} a$ that are loosely coupled to the B875 states, rather than transfer from higher-lying B875* states. These possibilities can be excluded by analysis of the waiting time dynamics. The cross peak between B875* and B875 grows in rapidly at early waiting times. A representative waiting time trace at $\lambda_{\tau}=770 \mathrm{~nm}$ and $\lambda_{t}=880 \mathrm{~nm}$ is shown in Figure 5. We observe that the waiting time trace reaches its maximum within $100 \mathrm{fs}$. When a biexponential function is fit to the data, the trace gives a fast rise of $31 \pm 3$ fs and a slow decay of $1280 \pm 100 \mathrm{fs}$ (see the SI for more details on the fitting procedure). Analysis of the complete cross peak region can be seen in Figure 5b. The rapid timescale for the growth of this cross peak excludes any possibility of energy transfer from free BChl $a$ to the B875 states, because any energy transfer between free BChl $a$ and LH1 would occur on a picosecond timescale due to weak coupling.

Fourier analysis in the waiting time domain is further utilized to elucidate the origin of the cross peaks. In 2DES, vibrational states give clear oscillations in the waiting time domain that result from vibrational coherences. ${ }^{31-33}$ A vibrational coherence is a superposition between two vibrational states within the same electronic excited state. In phased 2DES spectra, we observe excited state absorption features in the upper off-diagonal region of the 
map (Figure 3). From the waiting time trace, we see clear oscillations from this region that persist for more than $2 \mathrm{ps}$ (Figure 6a), which is on the appropriate timescale for vibrational coherences at room temperature. Fourier transforming the residual from exponential fitting yields four distinct vibrational modes at $96,288,558$, and $728 \mathrm{~cm}^{-1}$ (Figure 6b). These vibrational modes are also observed in the Raman spectrum of LH1 and free BChl a. ${ }^{15,34-36}$ When the cross peak between B875* and B875 is analyzed, no clear oscillations indicative of vibrational coherences can be resolved (Figure $6 \mathrm{c}$ and d). Thus, we attribute the cross peak on the lower off-diagonal region to be energy transfer from higher-lying B875* states to lower-lying B875 states.

Redfield theory was further used to validate the rapid energy transfer rates observed from B875* to B875. The sub-100 fs transfer rate is similar to results obtained in LH2 from B850* to B850. ${ }^{37}$ The rapid energy transfer is largely due to the co-localization of the excitations on the same ring of chromophores. Figure S8 shows the transition dipole weight for the $\mathrm{B} 875$ and $\mathrm{B} 875^{*}$ states for the three Hamiltonians. In addition to spatially overlapping states, the rapid rate of energy transfer is also due to high-energy bath modes, some of which can be seen in the vibrational coherences in Figures 6 and S9. In order to properly weight these high-energy bath modes, we used a log-normal spectral density given by

$$
J(\omega)=\frac{s}{\sigma \omega \sqrt{2 \pi}} e^{-\left[\operatorname{In}\left(\frac{\omega}{\omega_{c}}\right)\right] / 2 \sigma^{2}},
$$

with a high cutoff frequency, $\omega_{\mathcal{C}}$ of $1000 \mathrm{~cm}^{-1} .38$ The Huang-Rhys factor, $S$, was 0.5 and the standard deviation, $\sigma$, was $0.8 \mathrm{~cm}^{-1}$ Using this spectral density, the population transfer time was calculated following the procedure outlined by M. Reppert. ${ }^{39}$ The lifetime for $\mathrm{H}_{\text {closed }}^{(15)}$, calculated as the transfer time from $\mathrm{k}_{ \pm 14}$ to $\mathrm{k} 0$ and $\mathrm{k}_{ \pm 1}$, was found to be $51 \mathrm{fs}$. Similarly, the

lifetime for $\mathrm{H}_{\text {closed }}^{(16)}$ calculated as the transfer time from $\mathrm{k}_{16}$ to $\mathrm{k}_{0}$ and $\mathrm{k}_{ \pm 1}$, was found to be 54 fs. Lastly, the lifetime for $\mathrm{H}_{\mathrm{open}}^{(14)}$, calculated as the transfer time from $\mathrm{E}_{28}$ to $\mathrm{E}_{1-3}$, was found to be $42 \mathrm{fs}$. All values are within a factor of two of the observed energy transfer lifetimes of 30-40 fs.

\section{Conclusion}

The rapid growth of the cross peak and lack of vibrational coherences in the waiting time frequency domain firmly assigns the B875* states as electronic in origin. Their rapid transfer to B875 mirrors the dynamics found in the B850* and B850 states of $\mathrm{LH} 2$ and is in agreement with the effective Hamiltonians and Redfield theory calculations of population transfer rates. The role of $\mathrm{B} 875^{*}$ remains uncertain. The energy of $\mathrm{B} 875^{*}$ and its proximity to the $\mathrm{RC}$ and $\mathrm{LH} 2$ raises the possibility of it being an intermediate state as energy is transferred from the peripheral antenna to the $\mathrm{RC}$, though this is unlikely. Due to the short lifetime of B875*, direct transfer to the RC would not compete with relaxation to the B875 ring despite the strong spectral overlap of the $\mathrm{H}$ and $\mathrm{B}$ peaks of the $\mathrm{RC}$ with the $\mathrm{B} 875^{*}$ 
states. Also, the distance between B800 and B875 makes energy transfer from B800 to B875* an unlikely pathway. While other mechanisms such as super-transfer or moderation of energy transfer based the state of the reaction center are conceivable, the presence of the B875* states is likely an informative byproduct of the strong coupling between chromophores in the B875 ring, which is essential for biological function.

\section{Supplementary Material}

Refer to Web version on PubMed Central for supplementary material.

\section{Acknowledgments}

The authors would like to thank M. Reppert for the time and effort he spent discussing the project and the underlying theory with the authors. The authors also thank MRSEC (DMR 14- 20709), the DARPA QuBE program (N66001-10-1-4060), AFSOR (FA9550-09-1-0117), the Camille and Henry Dreyfus Foundation, and the Searle Foundation for supporting the work in this publication. S.C.M. acknowledges support from the Department of Defense (DoD) through the National Defense Science \& Engineering Graduate Fellowship (NDSEG) Program. P.D.D. acknowledges support from the NSF-GFRP program, and the National Institute of Biomedical Imaging And Bioengineering of the National Institutes of Health under Award Number T32-EB009412. C.N.H and E.C.M. were supported by grant BB/M000265/1 from the Biotechnology and Biological Sciences Research Council (UK) and an Advanced Award from the European Research Council (338895). This research was also supported by the Photosynthetic Antenna Research Center (PARC), an Energy Frontier Research Center funded by the U.S. Department of Energy, Office of Science, Office of Basic Energy Sciences under Award Number DE-SC 0001035. That grant provided partial support for C.N.H.

\section{References}

1. Blankenship, RE. Molecular Mechanisms of Photosynthesis. Blackwell Science; Oxford ; Malden, MA: 2002.

2. Amerongen, Hv, Valkunas, L., Grondelle, Rv. Photosynthetic Excitons. World Scientific; Singapore ; River Edge, N.J: 2000.

3. Wraight CA, Clayton RK. The Absolute Quantum Efficiency of Bacteriochlorophyll Photooxidation in Reaction Centres of Rhodopseudomonas sphaeroides. Biochim Biophys Acta, Bioenerg. 1974; 333:246-260.

4. Qian P, Papiz MZ, Jackson PJ, Brindley AA, Ng IW, Olsen JD, Dickman MJ, Bullough PA, Hunter CN. Three-Dimensional Structure of the Rhodobacter sphaeroides RC-LH1-PufX Complex: Dimerization and Quinone Channels Promoted by PufX. Biochemistry. 2013; 52:7575-7585. [PubMed: 24131108]

5. Qian P, Bullough PA, Hunter CN. Three-Dimensional Reconstruction of a Membrane-Bending Complex - the RC-LH1-PufX Core Dimer of Rhodobacter sphaeroides. J Biol Chem. 2008; 283:14002-14011. [PubMed: 18326046]

6. Sener M, Hsin J, Trabuco LG, Villa E, Qian P, Hunter CN, Schulten K. Structural Model and Excitonic Properties of the Dimeric RC-LH1-PufX Complex from Rhodobacter sphaeroides. Chem Phys. 2009; 357:188-197. [PubMed: 20161332]

7. Damjanovic A, Ritz T, Schulten K. Excitation Energy Trapping by the Reaction Center of Rhodobacter sphaeroides. Int J Quantum Chem. 2000; 77:139-151.

8. Visscher KJ, Bergstrom H, Sundstrom V, Hunter CN, Vangrondelle R. Temperature-Dependence of Energy-Transfer from the Long Wavelength Antenna BChl-896 to the Reaction Center in Rhodospirillum rubrum, Rhodobacter sphaeroides (WT and M21 Mutant) from 77 to 177K, Studied by Picosecond Absorption-Spectroscopy. Photosynth Res. 1989; 22:211-217. [PubMed: 24424811]

9. Beekman LMP, Vanmourik F, Jones MR, Visser HM, Hunter CN, Vangrondelle R. Trapping Kinetics in Mutants of the Photosynthetic Purple Bacterium Rhodobacter sphaeroides - Influence of the Charge Separation Rate and Consequences for the Rate-Limiting Step in the Light-Harvesting Process. Biochemistry. 994; 33:3143-3147. [PubMed: 8136347] 
10. Bahatyrova S, Frese RN, van der Werf KO, Otto C, Hunter CN, Olsen JD. Flexibility and Size Heterogeneity of the LH1 Light Harvesting Complex Revealed by Atomic Force Microscopy Functional Significance for Bacterial Photosynthesis. J Biol Chem. 2004; 279:21327-21333. [PubMed: 14993213]

11. Olsen JD, Adams PG, Jackson PJ, Dickman MJ, Qian P, Hunter CN. Aberrant Assembly Complexes of the Reaction Center Light-Harvesting 1 PufX (RC-LH1-PufX) Core Complex of Rhodobacter sphaeroides Imaged by Atomic Force Microscopy. J Biol Chem. 2014; 289:2992729936. [PubMed: 25193660]

12. Jamieson SJ, Wang PY, Qian P, Kirkland JY, Conroy MJ, Hunter CN, Bullough PA. Projection Structure of the Photosynthetic Reaction Centre- Antenna Complex of Rhodospirillum rubrum at 8.5 Angstrom Resolution. EMBOJ. 2002; 21:3927-3935.

13. Bradforth SE, Jimenez R, Vanmourik F, Vangrondelle R, Fleming GR. Excitation Transfer in the Core Light-Harvesting Complex (LH1) of Rhodobacter sphaeroides - an Ultrafast Fluorescence Depolarization and Annihilation Study. J Phys Chem. 1995; 99:16179-16191.

14. Jimenez R, van Mourik F, Yu JY, Fleming GR. Three-Pulse Photon Echo Measurements on LH1 and LH2 Complexes of Rhodobacter sphaeroides: A Nonlinear Spectroscopic Probe of Energy Transfer. J Phys Chem B. 1997; 101:7350-7359.

15. Chachisvilis M, Pullerits T, Jones MR, Hunter CN, Sundstrom V. Vibrational Dynamics in the Light-Harvesting Complexes of the Photosynthetic Bacterium Rhodobacter sphaeroides. Chem Phys Lett. 1994; 224:345-351.

16. Stuart TAC, Vengris M, Novoderezhkin VI, Cogdell RJ, Hunter CN, van Grondelle R. Direct Visualization of Exciton Reequilibration in the LH1 and LH2 Complexes of Rhodobacter sphaeroides by Multipulse Spectroscopy. Biophys J. 2011; 100:2226-2233. [PubMed: 21539791]

17. Pullerits T, Chachisvilis M, Jones MR, Hunter CN, Sundström V. Exciton Dynamics in the LightHarvesting Complexes of Rhodobacter sphaeroides. Chem Phys Lett. 1994; 224:355-365.

18. Mothersole DJ, Jackson PJ, Vasilev C, Tucker JD, Brindley AA, Dickman MJ, Hunter CN. PucC and LhaA Direct Efficient Assembly of the Light-Harvesting Complexes in Rhodobacter sphaeroides. Mol Microbiol. 2016; 99:307-327. [PubMed: 26419219]

19. Brixner T, Stenger J, Vaswani HM, Cho M, Blankenship RE, Fleming GR. Two-Dimensional Spectroscopy of Electronic Couplings in Photosynthesis. Nature. 2005; 434:625-628. [PubMed: 15800619]

20. Hybl JD, Albrecht Ferro A, Jonas DM. Two-Dimensional Fourier Transform Electronic Spectroscopy. J Chem Phys. 2001; 115:6606-6622.

21. Hybl JD, Albrecht AW, Faeder SMG, Jonas DM. Two-Dimensional Electronic Spectroscopy. Chem Phys Lett. 1998; 297:307-313.

22. Mukamel, S. Principles of Nonlinear Optical Spectroscopy. Oxford University Press; New York; Oxford: 1995.

23. Hamm, P., Zanni, MT. Concepts and Methods of 2D Infrared Spectroscopy. Cambridge University Press; Cambridge ; New York: 2011.

24. Brixner T, Mancal T, Stiopkin IV, Fleming GR. Phase-Stabilized Two- Dimensional Electronic Spectroscopy. J Chem Phys. 2004; 121:4221-36. [PubMed: 15332970]

25. Harel E, Fidler AF, Engel GS. Real-Time Mapping of Electronic Structure with Single-Shot TwoDimensional Electronic Spectroscopy. Proc Natl Acad Sci U S A. 2010; 107:16444-16447. [PubMed: 20810917]

26. Harel E, Long PD, Engel GS. Single-Shot Ultrabroadband Two- Dimensional Electronic Spectroscopy of the Light-Harvesting Complex LH2. Opt Lett. 2011; 36:1665-1667. [PubMed: 21540962]

27. Harel E, Fidler AF, Engel GS. Single-Shot Gradient-Assisted Photon Echo Electronic Spectroscopy. J Phys Chem A. 2011; 115:3787-3796. [PubMed: 21090733]

28. Dahlberg PD, Fidler AF, Caram JR, Long PD, Engel GS. Energy Transfer Observed in Live Cells Using Two-Dimensional Electronic Spectroscopy. J Phys Chem Lett. 2013; 4:3636-3640. [PubMed: 24478821]

29. Singh VP, Fidler AF, Rolczynski BS, Engel GS. Independent Phasing of Rephasing and NonRephasing 2D Electronic Spectra. J Chem Phys. 2013; 139:084201. [PubMed: 24006987] 
30. Niwa S, Yu LJ, Takeda K, Hirano Y, Kawakami T, Wang-Otomo ZY, Miki K. Structure of the LH1RC Complex from Thermochromatium tepidum at 3.0 Angstrom. Nature. 2014; 508:228-232. [PubMed: 24670637]

31. Fransted KA, Engel GS. Probing Vibrational Dynamics of PM650 with Two-Dimensional Electronic Spectroscopy. Chem Phys. 2012; 403:59-67.

32. Fransted KA, Caram JR, Hayes D, Engel GS. Two-Dimensional Electronic Spectroscopy of Bacteriochlorophyll a in Solution: Elucidating the Coherence Dynamics of the Fenna-MatthewsOlson Complex Using Its Chromophore as a Control. J Chem Phys. 2012; 137:125101. [PubMed: 23020349]

33. Butkus V, Zigmantas D, Valkunas L, Abramavicius D. Vibrational vs Electronic Coherences in 2D Spectrum of Molecular Systems. Chem Phys Lett. 2012; 545:40-43.

34. Donohoe RJ, Frank HA, Bocian DF. Resonance Raman-Spectra and Normal Mode Descriptions of a Bacteriochlorophyll-a Model Complex. Photochem Photobiol. 1988; 48:531-537. [PubMed: 3266014]

35. Cherepy NJ, Shreve AP, Moore LJ, Boxer SG, Mathies RA. Temperature Dependence of the Q(Y) Resonance Raman Spectra of Bacteriochlorophylls, the Primary Electron Donor, and Bacteriopheophytins in the Bacterial Photosynthetic Reaction Center. Biochemistry. 1997; 36:8559-8566. [PubMed: 9214301]

36. Shreve AP, Cherepy NJ, Franzen S, Boxer SG, Mathies RA. Rapid-Flow Resonance RamanSpectroscopy of Bacterial Photosynthetic Reaction Centers. Proc Natl Acad Sci U S A. 1991; 88:11207-11211. [PubMed: 1763034]

37. Novoderezhkin V, Wendling M, van Grondelle R. Intra- and Interband Transfers in the B800-B850 Antenna of Rhodospirillum molischianum: Redfield Theory Modeling of Polarized Pump-Probe Kinetics. J Phys Chem B. 2003; 107:11534-11548.

38. Kell A, Feng X, Reppert M, Jankowiak R. On the Shape of the Phonon Spectral Density in Photosynthetic Complexes. J Phys Chem B. 2013; 117:7317-7323. [PubMed: 23718713]

39. Reppert M. Modeling of Resonant Hole-Burning Spectra in Excitonically Coupled Systems: The Effects of Energy-Transfer Broadening. J Phys Chem Lett. 2011; 2:2716-2721.

J Phys Chem A. Author manuscript; available in PMC 2017 November 02. 


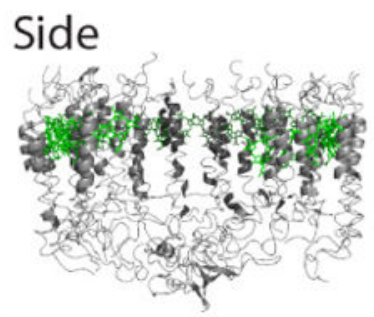

Top

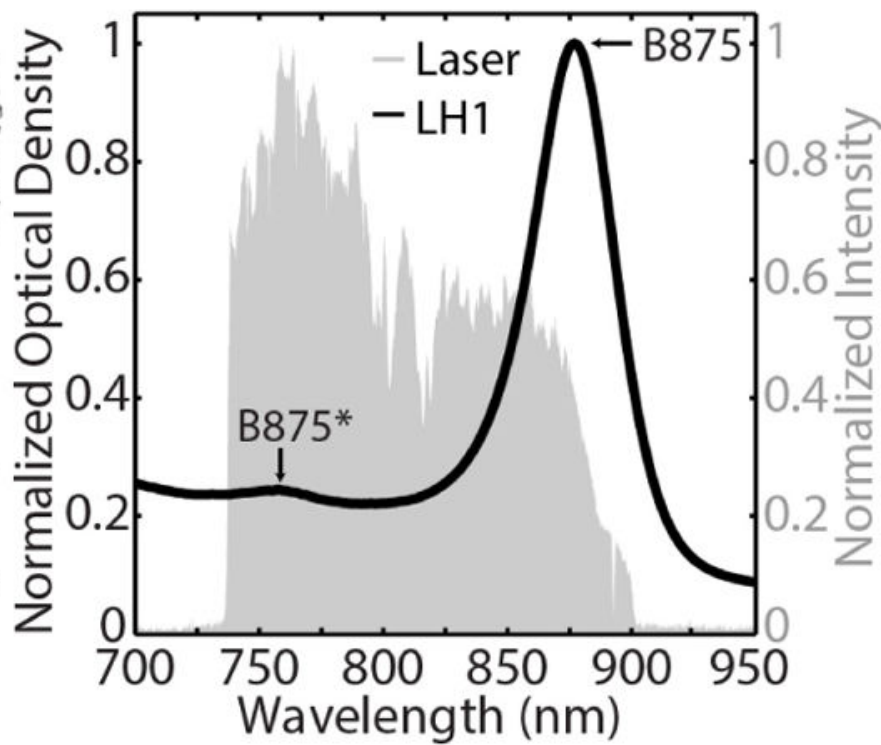

Figure 1.

Left: The $8 \AA$ A-resolution structure of a monomer of LH1 complex in R. sphaeroides reconstructed from the RC-LH1 dimeric crystal structure found in PDB ID: 4V9G. ${ }^{14}$ The protein backbone (shown in gray) holds $28 \mathrm{BChl}$ a (shown in green) tightly in space. Right. The absorption spectrum of LH1-only membranes is shown in black. The two visible features near $765 \mathrm{~nm}$ and $875 \mathrm{~nm}$ correspond to B875* and B875, respectively. The normalized laser excitation spectrum used for the 2DES experiments is shown in shaded gray. 

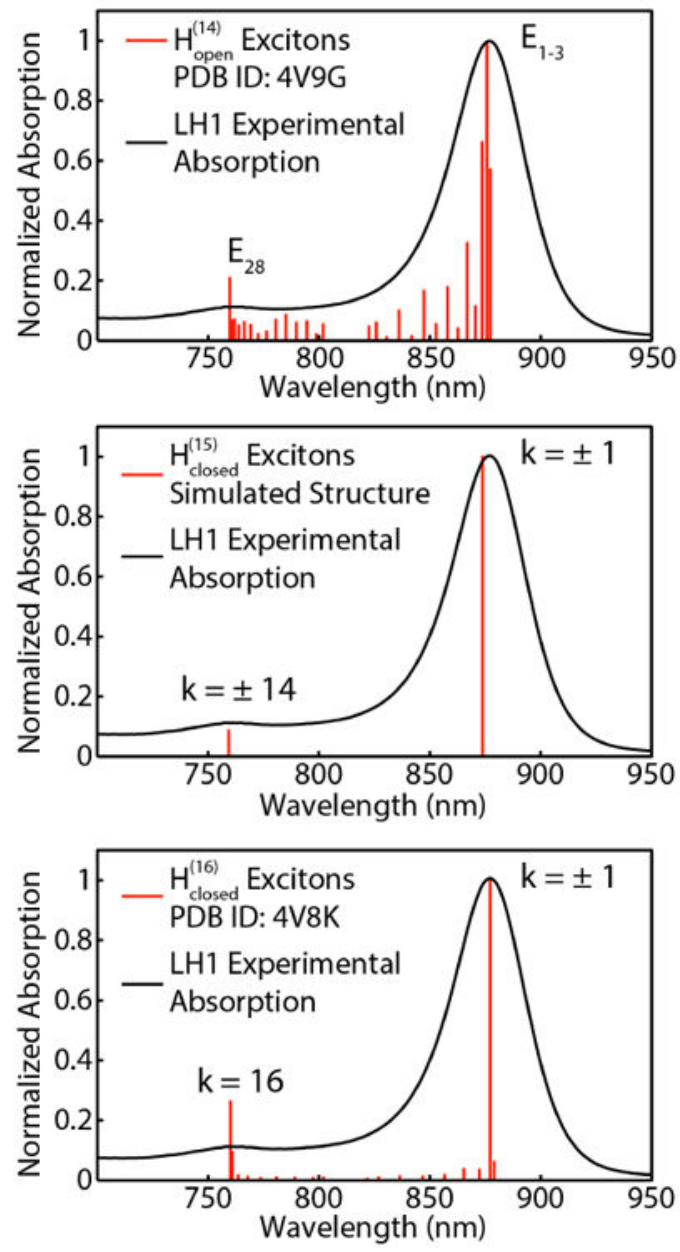

Figure 2.

Stick spectra from $\mathrm{H}_{\text {open }}^{(14)}, \mathrm{H}_{\text {closed }}^{(15)}$, and $\mathrm{H}_{\text {closed }}^{(16)}$ are overlaid with the absorption spectra of LH1-only membranes after the removal of Mie scatter. The stick spectra are in good agreement with the absorption spectra, recapitulating the strong B875 states and the weak B875* states. 

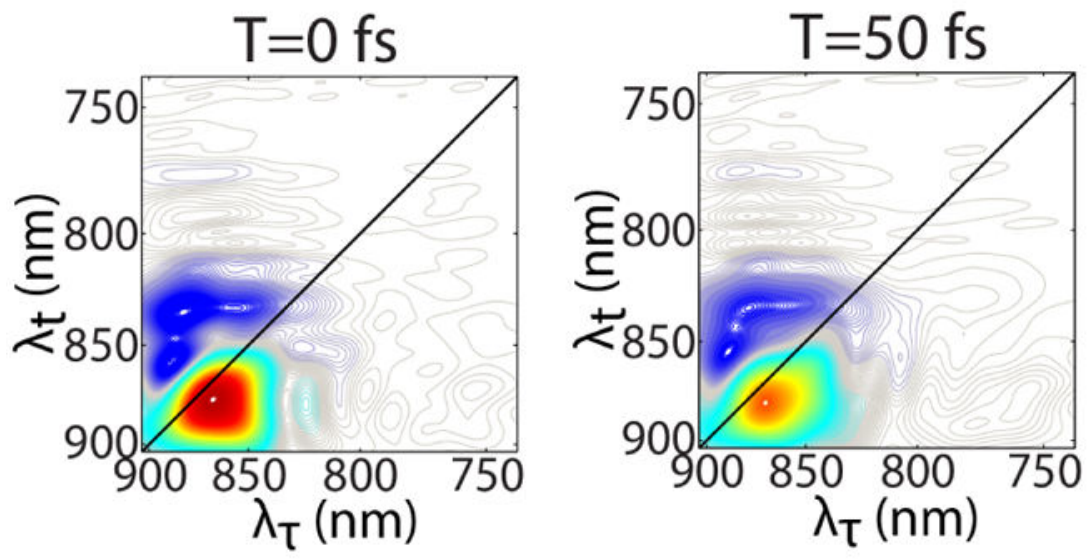

Amp.
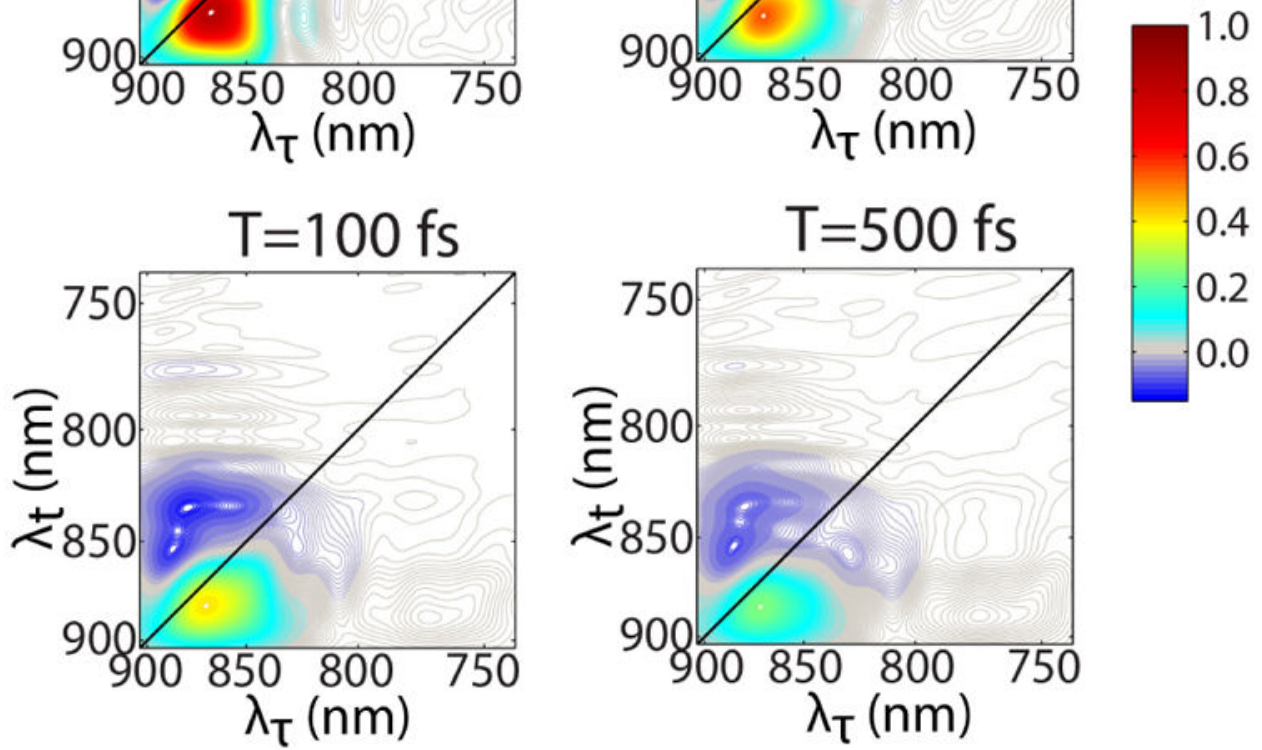

Figure 3.

Representative phased rephasing 2DES spectra of LH1-only membranes at waiting times of $\mathrm{T}=0 \mathrm{fs}, \mathrm{T}=50 \mathrm{fs}, \mathrm{T}=100 \mathrm{fs}$ and $\mathrm{T}=500 \mathrm{fs}$. All spectra are normalized to the signal maximum across all waiting times. 


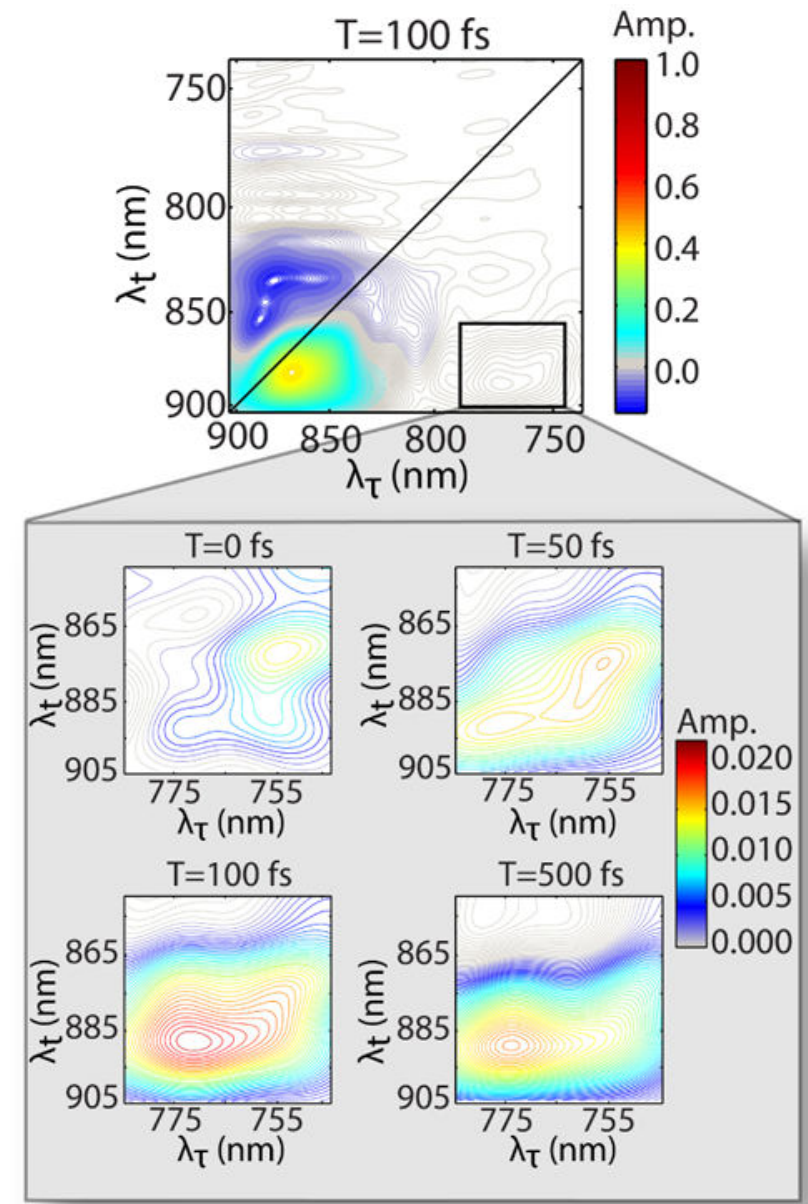

Figure 4.

Phased rephasing 2DES spectra of the cross peak between B875* and B875 (region enclosed by the red rectangle in the 2DES spectrum above) in LH1-only membranes showing stimulated emission around $\lambda_{\tau}=770 \mathrm{~nm}$ and $\lambda_{\mathrm{t}}=880 \mathrm{~nm}$ at waiting times of $\mathrm{T}=0 \mathrm{fs}, \mathrm{T}=$ $50 \mathrm{fs}, \mathrm{T}=100 \mathrm{fs}$ and $\mathrm{T}=500 \mathrm{fs}$, respectively. All spectra are normalized to the signal maximum across all waiting times. 


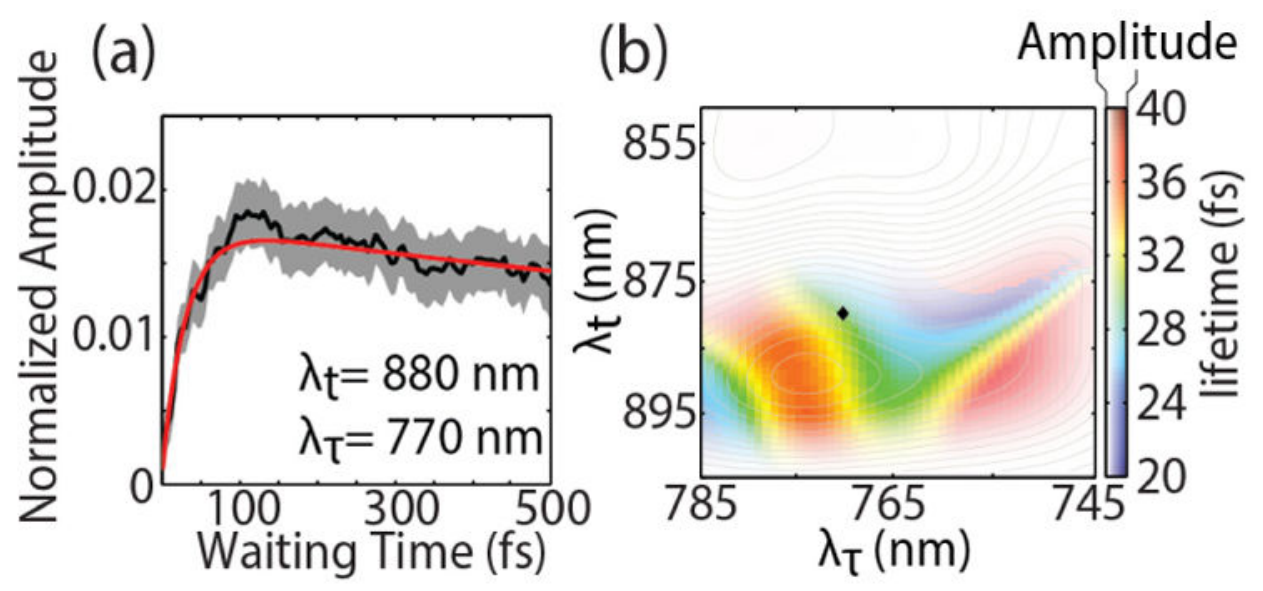

Figure 5.

Representative waiting time trace and lifetime map at the B875*-B875 cross peak of LH1only membranes show rapid energy transfer within $40 \mathrm{fs}$. (a) The black curve is the average waiting time trace of LH1-only membranes at $\lambda_{\tau}=770 \mathrm{~nm}$ and $\lambda_{t}=880 \mathrm{~nm}$ (indicated by black diamond in the spectrum to the right) of 10 scans acquired in rapid succession over the course of 2 hours. The width of the gray shading is the standard error $(1 \sigma)$ representing our uncertainty in the mean. The red trace is a regression of the data to a model bi-exponential function. (b) Lifetime map for the rapid component of the bi-exponential around $\lambda_{\tau}=770$ $\mathrm{nm}$ and $\lambda_{\mathrm{t}}=880 \mathrm{~nm}$. The saturation level of the map is weighted by both the signal strengths at $\mathrm{T}=500$ fs for each pixel. The contour curves represent the phased 2DES spectrum at waiting time of $100 \mathrm{fs}$. 
(a)
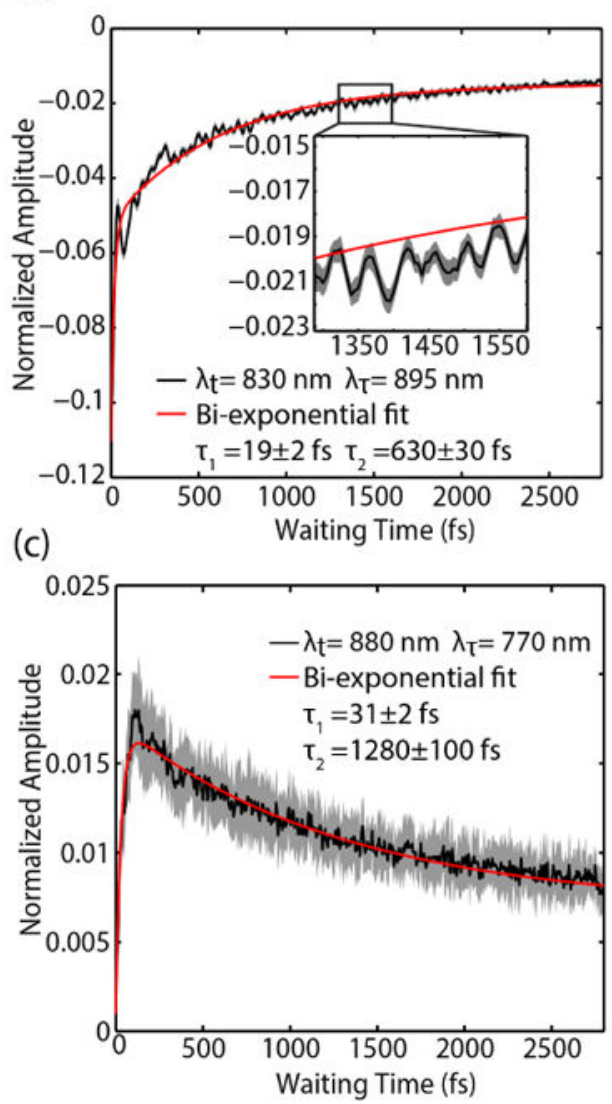

(b)

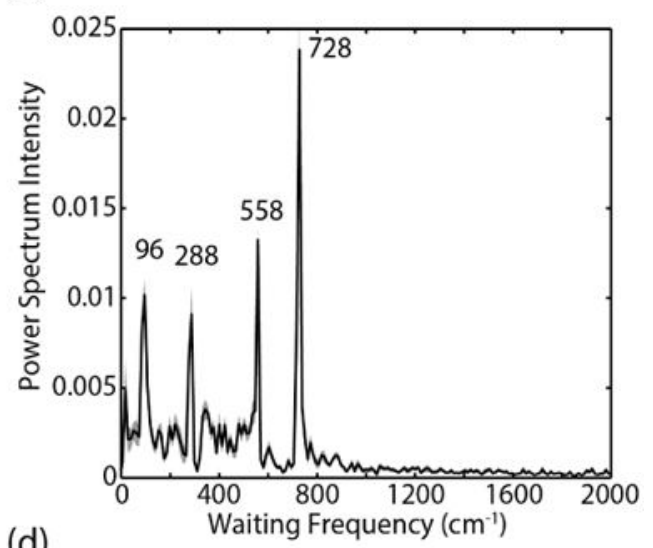

(d)

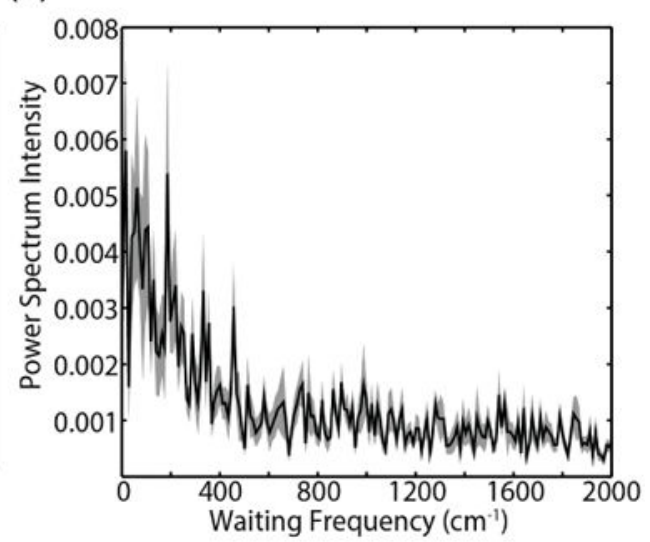

Figure 6.

Waiting time traces from two locations in the 2DES spectra and their conjugate waiting time frequency traces show no clear vibrations at the cross peak between B875* and B875. All black traces in the figures represent an average of 10 scans. The width of the gray shading is the standard error $(1 \sigma)$ representing our uncertainty in the mean. (a) Waiting time trace of LH1-only membranes at $\lambda_{\tau}=895 \mathrm{~nm}$ and $\lambda_{\mathrm{t}}=830 \mathrm{~nm}$ showing oscillations due to vibrational coherences at this location. (b) Average power spectrum of the residuals from fitting the same waiting time trace in (a) for each of the 10 scans (resolution is $11 \mathrm{~cm}^{-1}$ ). These peaks are known vibrational modes of BChl $a .{ }^{34}$ (c) Waiting time trace from the $\mathrm{B} 875^{*}$-B875 lower cross peak at $\lambda_{\tau}=770 \mathrm{~nm}$ and $\lambda_{\mathrm{t}}=880 \mathrm{~nm}$. (d) Average power spectrum of the residuals from fitting the same waiting time trace in (c) for each of the 10 scans. 